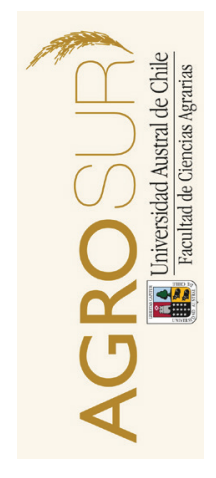

\title{
Efecto del flujo de agua y el volumen de muestreo sobre la funcionalidad de los poros de un Ultisol
}

\author{
Effect of water flow and sampling volume \\ on the functional characterization of pores in an Ultisol
}

\author{
Leiva, C. ${ }^{a}$, Dec, D. ${ }^{a, b}$, Seguel, 0. ${ }^{b, c}$, Nissen, J. ${ }^{a}$, Dörner, J. ${ }^{a, b *}$ \\ ${ }^{a}$ Instituto de Ingeniería Agraria y Suelos, Universidad Austral de Chile. Casilla 567, Valdivia, Chile. \\ ${ }^{b}$ Centro de Investigación en Suelos Volcánicos, Universidad Austral de Chile. Casilla 567, Valdivia, Chile. \\ ${ }^{c}$ Departamento de Ingeniería y Suelos, Facultad de Ciencias Agronómicas, Universidad de Chile, \\ casilla 1004, Santiago, Chile.
}

\begin{tabular}{l} 
A R T I C L E I N F O \\
\hline Keywords: \\
Typic Hapludult \\
Air permeability \\
Hydraulic conductivity \\
Pore functions \\
Soil structure \\
\hline Original Research Article, \\
Soil Science \\
\hline${ }^{*}$ Corresponding author: \\
César Leiva, José Dörner \\
E-mail address: \\
josedorner@uach.cl
\end{tabular}

\begin{abstract}
A B S T R A C T
The porous system controls the movement of fluids and its description must consider its volume, distribution and functionality. The aim of this work was to evaluate the functional heterogeneity of the pore system of a volcanic ash soil, considering both the sampling volume and the changes in soil structure under conditions of saturated flow. The pore system of an Ultisol (Cudico soil Series) was characterized using non-disturbed samples collected in cylinders of 110 and $250 \mathrm{~cm}^{3}$. The water retention curve, air filled-porosity $\left(\varepsilon_{a}\right)$, air permeability $\left(k_{a}\right)$, saturated hydraulic conductivity (kf), pore continuity and the volume of blocked pores $\left(\varepsilon_{b}\right)$ were determined. Besides, it was evaluated how the amount and functionality of the porous system is affected by a constant flow of water for $24 \mathrm{~h}$. When comparing both sampling volumes, statistical differences were found in the capacity of the soil to retain water and in $\varepsilon_{a}$ at water tension lower than $60 \mathrm{hPa}$. The saturated flow of water generated a loss in the drainage pores, a decrease of $k_{a}$ and an increase in the number of blocked pores and the continuity index $k_{2}$. Therefore, the conditions of saturated water flow alter the soil structure affecting the functionality of the pore system, being this factor more relevant than the volume of the sample when characterizing the pore functionality of the soil.
\end{abstract}

\section{RESUMEN}

El sistema poroso controla el movimiento de fluidos y su descripción debe realizarse considerando su volumen, distribución y funcionalidad. El objetivo de este trabajo fue analizar la heterogeneidad funcional de los poros de un suelo derivado de cenizas volcánicas, considerando distintos volúmenes de muestreo y cambios en la estructura por flujo saturado de agua. Se caracterizó el sistema poroso de un Ultisol de la Serie Cudico, colectando muestras sin disturbar en cilindros de 110 y $250 \mathrm{~cm}^{3}$. Se determinó la curva de retención de agua, la fracción de poros llenos de aire $\left(\varepsilon_{a}\right)$, la permeabilidad de aire $\left(k_{a}\right)$, la conductividad hidráulica saturada $(\mathrm{kf})$, la continuidad de poros y el volumen de poros bloqueados $\left(\varepsilon_{b}\right)$. Se evaluó cómo es afectado el monto y la funcionalidad del sistema poroso luego de un flujo constante de agua durante $24 \mathrm{~h}$. Al comparar ambos volúmenes de muestreo, se encontraron diferencias en la capacidad del suelo de retener agua $\left(\mathrm{Cil}_{110 \mathrm{~cm}}{ }^{3}<\mathrm{Cil}_{250 \mathrm{~cm}}{ }^{3}\right)$ y en la porosidad con aire $\left(\mathrm{Cil}_{110 \mathrm{~cm}}{ }^{3}>\mathrm{Cil}_{250 \mathrm{~cm}}{ }^{3}\right)$ a tensiones menores a $60 \mathrm{hPa}$. La permeabilidad al aire, que no presentó diferencias entre volúmenes de muestreo. El flujo saturado de agua generó una pérdida en los poros de drenaje, una disminución de $k_{a}$ y un aumento en la cantidad de poros bloqueados y del índice de continuidad $k_{2}$. Las condiciones de flujo saturado de agua alteran la estructura del suelo, afectando la funcionalidad del sistema poroso, siendo un factor más relevante que el volumen de la muestra al caracterizar la funcionalidad porosa del suelo.

Palabras clave: Typic Hapludult, permeabilidad al aire, conductividad hidráulica, funcionalidad de poros, estructura de suelo.

\section{INTRODUCCIÓN}

La descripción de la geometría del sistema poroso y su funcionalidad en el suelo está relacionada con la distribución del tamaño de sus partículas y su estructura (Rawls y Pachepsky, 2002). Por lo tanto, la porosidad del suelo puede estar formada por una porosidad textural, que da origen a una distribución secuencial de poros en el suelo (Hunt, 2005). Estos poros son afectados en menor medida por la labranza y el manejo del suelo (Hartge y Horn, 2009). Por otro lado, la estructura del suelo, se define como la disposición de partículas minerales y sustancias orgánicas en unidades mayores conocidas como agregados, que incluyen poros entre los agregados (Horn y Smucker, 2005). Esta porosidad de carácter estructural comprende grietas, bio-poros 
y macro estructura, las cuales son sensibles a factores tales como la labranza, la compactación y los ciclos de mojado y secado (Dexter, 2004; Horn y Smucker, 2005; Dörner et al., 2012). Por lo tanto, la cantidad, tamaño, forma y continuidad de los poros son propiedades usadas en la caracterización de la estructura del suelo (Dörner y Horn, 2006; Pagenkemper et al., 2014).

El volumen total del sistema poroso del suelo está constituido por una fase gaseosa y una fase líquida, cambiando continuamente su proporción debido a procesos de humectación y secado, agregación y dispersión, entre otros (Hillel, 1982). Producto de esta versatilidad se utiliza el concepto de volumen elemental representativo (REV, representative elemental volumen, sigla en inglés), desarrollado para encontrar el volumen mínimo de muestreo que represente estadísticamente la variabilidad del medio poroso. El REV depende del análisis a llevar a cabo y, en términos generales, cuando se quiere caracterizar aspectos funcionales de los poros del suelo debe ser superior a $200 \mathrm{~cm}^{3}$ (Hartge y Horn, 2009). Por lo tanto, al colectar volúmenes menores aumenta la variabilidad entre muestras y predominan los efectos de la micro escala en la medición, mientras que al extraer volúmenes más grandes disminuye la capacidad de procesamiento de ellas (Hunt, 2005; Dietrich et al., 2005; Vafai, 2005; Clifford y Stephen, 2006). Lo anterior es relevante debido a que un inadecuado REV puede implicar un incorrecta determinación de volumen de agua aprovechable para las plantas o una incorrecta determinación de la conductividad hidráulica saturada, parámetros importantes para tomar decisiones de tipo agronómicas y también para estudios ambientales (Hartge y Horn, 2009).

El sistema poroso del suelo es la ruta por donde fluye el agua y el aire. La continuidad vertical a través de los horizontes del suelo es relevante para transportar el agua e intercambiar el gas atmosférico (Lal et al., 2004). En un suelo mojado que se empieza a secar, el flujo de agua disminuye pero el flujo de aire aumenta (Dietrich et al., 2005); por su parte, procesos largos de humectación generan hinchamientos del suelo que podrían causar cambios en la distribución y continuidad porosa, especialmente en aquellos con altos niveles de arcilla (Fuentes et al., 2015). Por lo tanto, la caracterización del espacio poroso es fundamental en la determinación del movimiento de fluidos en el suelo (Roseberg y McCoy, 1990; Iversen et al., 2001; Dörner y Horn, 2006). En ese contexto, el movimiento de fluidos en el suelo es primordial para la comprensión de procesos de transporte, como por ejemplo la dinámica del agua en el suelo. Esto último es importante no tan sólo desde el punto del uso eficiente de agua en la agricultura (p. ej. Noellemeyer et al., 2013), sino que también por la implicancia ambiental que ciertas condiciones de flujo pueden provocar sobre la calidad de las aguas freáticas (p. ej. Hopmans y van Genuchten, 2005). Además, car- gas continuas sobre el suelo como el pisoteo animal y uso excesivo de maquinaria, produce la compactación del suelo redistribuyendo el volumen poroso, disminuyendo la capacidad de infiltración y de almacenamiento de agua en el suelo (Hartge y Horn, 1991; Hillel, 1998).

Dados estos antecedentes, es posible establecer que existen dos elementos relevantes a considerar en la evaluación de medios porosos: $i$ ) la determinación del volumen de suelo que represente la complejidad de la estructura y el funcionamiento de los poros y, ii) cómo las condiciones de flujo se pueden ver afectadas por potenciales cambios en la distribución de poros bajo condiciones saturadas de flujo. Por lo tanto, los objetivos de este trabajo fueron $i$ ) determinar si el volumen de muestreo de $110 \mathrm{~cm}^{3}$ representa la heterogeneidad funcional de los poros de un Ultisol caracterizada a partir de muestras $250 \mathrm{~cm}^{3} \mathrm{y}$, ii) evaluar el efecto del flujo saturado de agua sobre el monto y funcionalidad de los poros del suelo.

\section{MATERIALES Y MÉTODO}

\section{Sitio de muestreo}

Para este estudio se colectaron muestras de un predio ubicado a $3,5 \mathrm{~km}$ de la ciudad de La Unión $\left(40^{\circ} 18^{\prime} 33^{\prime \prime} \mathrm{S}-73^{\circ} 06^{\prime} 45^{\prime \prime} 0\right.$; $\left.30 \mathrm{msnm}\right)$, realizando un muestreo de 0 a $20 \mathrm{~cm}$ de profundidad en un suelo bajo pradera artificial dominada por Lolium perenne L. El suelo corresponde a un Typic Hapludult, Serie Cudico, formado a partir de depósitos antiguos de cenizas volcánicas sobre sedimentos marinos. Son suelos moderadamente profundos, de colores pardo oscuro en superficie a pardo rojizo en profundidad (CIREN, 2003). En una caracterización previa realizada por Leiva (2009), se determinó que la distribución del tamaño de las partículas del suelo presenta un alto contenido de arena $(20,09 \%)$, limo $(37,17 \%)$ y arcilla $(42,74 \%)$. Además, es un suelo bien estructurado, firme, plástico y adhesivo (CIREN, 2003). Las condiciones climáticas del lugar destacan por una temperatura media anual de entre 11 y $12{ }^{\circ} \mathrm{C}$, con mínimas en julio $\left(4,6^{\circ} \mathrm{C}\right.$ promedio $)$ y máximas en enero $\left(21,9^{\circ} \mathrm{C}\right.$ promedio), precipitación anual cercana a $2.000 \mathrm{~mm}$ y ETP que varía entre 530 y 660 $\mathrm{mm}$ al año (Uribe et al., 2012).

\section{Análisis de laboratorio}

Las muestras no disturbadas fueron recolectadas en cilindros metálicos de $110 \mathrm{~cm}^{3}$ (diámetro $=6,9 \mathrm{~cm}$, alto $=3 \mathrm{~cm}$ ) y $250 \mathrm{~cm}^{3}$ (diámetro $=7,99 \mathrm{~cm}$, alto $=4,98$ $\mathrm{cm}$ ), denominados respectivamente: $\mathrm{Cil}_{110 \mathrm{~cm}} 3 \mathrm{y} \mathrm{Cil}_{250 \mathrm{~cm}} 3$.

Para la determinación de la curva de retención de agua, los cilindros de suelo fueron saturados $(0 \mathrm{hPa})$ mediante ascenso capilar de agua con el objetivo de evitar inclusiones de aire en los poros (Hartge y Horn, 2009). 
Posteriormente, las muestras fueron equilibradas a potenciales mátricos de $-10,-20,-30$ y $-60 \mathrm{hPa}$ en bandejas de arena y $-150,-300$ y $-500 \mathrm{hPa}$ por medio de platos cerámicos en una olla de presión. La retención de agua en punto de marchitez permanente (tensión de 15.430 $\mathrm{hPa}$ ) se realizó con una muestra disturbada en plato de presión. Una vez que las muestras alcanzaron un equilibrio con el potencial mátrico aplicado, se determinó el contenido gravimétrico de agua por medio de pesaje (balanza Precisa, 0,01 g de precisión). Finalmente, las muestras fueron secadas a $105^{\circ} \mathrm{C}$ por 24 horas para determinar la densidad aparente y calcular los contenidos volumétricos de agua que permitieron estimar la distribución de tamaño de poros (Hartge y Horn, 2009).

La conductividad del aire $(k)$ se midió en un flujómetro de aire a potenciales mátricos de -30 -60, -150, -330 y -500 hPa por medio de un método de un flujo constante de aire en cámara colgante (Leiva, 2009) en ambos tamaños de cilindros. Para el correcto análisis de los resultados, se registraron las variaciones en la presión atmosférica y temperatura, valores que son considerados en los cálculos (Dörner y Dec, 2007). A partir de la conductividad de aire, se calculó la permeabilidad de aire a distintas tensiones, determinados con 14 cilindros de $250 \mathrm{~cm}^{3}$, independientes a los utilizados para la caracterización del sistema poroso, utilizando la siguiente ecuación:

$$
k_{a}\left(\varepsilon_{a}\right)=k_{l}\left(\varepsilon_{a}\right) * \frac{\eta}{\rho_{l} * g}
$$

Donde: $k_{a}=$ permeabilidad de aire $\left(\mu \mathrm{m}^{2}\right) \eta=$ es la viscosidad del aire $\left(\mathrm{g} \mathrm{cm}^{-1}\right) ; \rho_{l}=$ es la densidad del aire $\left(\mathrm{kg} \mathrm{m}^{-3}\right) ; g=$ es la aceleración de gravedad $\left(\mathrm{m} \mathrm{s}^{-2}\right)$ y $k_{l}$ es la conductividad de aire evaluada a los distintos equilibrios mátricos, por lo que es función de la porosidad con aire $\left(\varepsilon_{a}\right)$.

La determinación de la conductividad hidráulica saturada (kf) se realizó mediante un permeámetro de carga constante (Eijkelkamp), con las muestras estructuradas solo de $250 \mathrm{~cm}^{3}$, con mediciones en intervalos de 1, 8, 12 y 24 horas de comenzado el flujo. Posteriormente se volvió a medir la distribución de tamaño de poros, de manera de determinar si existen cambios en $k_{a}$ y en el espacio poroso por efecto de un flujo constante de agua a través del suelo.

\section{Determinación de parámetros de continuidad de medios porosos}

La continuidad del sistema poroso fue evaluada a partir de los índice $k_{1}$ y $k_{2}$ que relacionan la permeabilidad de aire y los poros llenos de aire $\left(\varepsilon_{a}\right)$ tal como lo hicieron Ball et al. (1988); Dörner y Horn (2006) y Dörner y Dec (2007). Estos índices son:

$$
\begin{gathered}
k_{1}=\frac{k_{a}}{\varepsilon_{a}} \\
k_{2}=\frac{k_{a}}{\left(\varepsilon_{a}\right)^{2}}
\end{gathered}
$$

donde $k_{1}$ y $k_{2}$ caracterizan la capacidad que tiene el suelo de transmitir aire. Al comparar ambos índices, estos deberían presentar valores análogos, de no ser así, significa que el suelo presenta diferencias en la continuidad del sistema poroso; estos índices pueden ser utilizados independiente de la distribución que presente el espacio poroso (Ball et al., 1988).

Ahuja et al. (1984) y Ball et al. (1988) describen otros índices de geometría del medio poroso derivados de la relación entre $k_{a}$ y $\varepsilon_{a^{\prime}}$ estableciendo:

$$
\log k_{a}=\log M+N \log \varepsilon_{a}
$$

donde $M$ y $N$ son parámetros empíricos del ajuste lineal entre $\varepsilon_{a}$ y $k_{a}$ en una escala doble logarítmica. $N$ es un índice de continuidad de poros que refleja el incremento de la permeabilidad del aire en función del aumento de los poros con aire (Ahuja et al., 1984; Ball et al., 1988), por lo que representa la disminución de la tortuosidad de los poros y del área superficial con el incremento de la fracción de poros disponibles para el flujo. Un índice que permite estimar el volumen de poros bloqueados $\left(\varepsilon_{b}\right)$ que no participan del flujo de aire por convección es propuesto por Ball et al. (1988). Los autores mencionan que suelos que presentan una permeabilidad de aire igual o menor a $1 \mu \mathrm{m}^{2}$ pueden ser considerados como impermeables; por lo tanto, el intercepto en la abscisa corresponde a un estimador de $\varepsilon_{b}$, tal que:

$$
\varepsilon_{b}=10^{\frac{(-M)}{N}}
$$

\section{Análisis estadístico}

Para establecer si a partir de una disminución del volumen de muestreo es posible representar la heterogeneidad del suelo, se procedió a comparar si existen diferencias estadísticas entre muestras recolectadas en cilindros de $\mathrm{Cil}_{110 \mathrm{~cm}^{3}}$ y $\mathrm{Cil}_{250 \mathrm{~cm}}$. Este último, se utilizó de referencia para compararlo en variables cuantitativas, tanto en el monto como en la funcionalidad del sistema poroso (Bartoli et al., 2005). Los resultados fueron sometidos a un análisis de varianza de dos vías (tamaño cilindro, tensión mátrica) con ocho repeticiones por tratamiento. Se realizaron las prueba de normalidad de Lilliefors y el test de homogeneidad de varianza de Cochran. En el caso que los valores no presentaron una distribución normal, estos fueron transformados a logaritmo, tal como lo plantea Hartge y Horn 
(2009). Luego se realizó un ANDEVA, y cuando existieron diferencias estadísticas significativas ( $\mathrm{p} \leq 0,05)$, se separaron las medias usando el test de LSD. Para realizar los análisis estadísticos se utilizó el complemento estadístico para Excel XLSTAT y el programa estadístico STATISTICA.

\section{RESULTADOS Y DISCUSIÓN}

\section{Caracterización del espacio poroso en relación al volumen de la muestra}

La porosidad total del suelo es alta (Cuadro 1) con un alto volumen de agua retenida que no puede ser utilizada por las plantas (punto de marchitez permanente o agua retenida a $\mathrm{pF} 4,19$ ), corroborando la estrecha relación entre los poros de agua inútil y la clase textural del suelo (Saxton y Rawls, 2003).

Al comparar los dos volúmenes de suelo, se aprecia que existen diferencias significativas a lo largo de todas las tensiones hasta $-500 \mathrm{hPa}$, pero no a la tensión de $15.430 \mathrm{hPa}$. Sin embargo, aunque los coeficientes de variación (CV) fueron más bajos en las muestras colectadas con cilindros $250 \mathrm{~cm}^{3}$, no se encontraron diferencias estadísticas entre los CV promedio en las distintas tensiones evaluadas, de los volúmenes de muestreo $\left(2,17\right.$ para $\mathrm{Cil}_{110 \mathrm{~cm}^{3}}$ y 1,39 para $\left.\mathrm{Cil}_{250 \mathrm{~cm}}{ }^{3}\right)$. En ese contexto, Bartoli et al. (2005) indican que las diferencias entre los CV entregan una buena aproximación de la representatividad del volumen de suelo, por lo tanto, al no existir diferencias entre ellos para el presente es- tudio, las diferencias encontradas entre los contenidos de agua de los dos volúmenes de suelo podrían deberse más a una característica de este que a un problema asociado al volumen de muestreo, debido a que la altura de los cilindros de $110 \mathrm{~cm}^{3}$ es cercana a $3 \mathrm{~cm}$ y las de 250 $\mathrm{cm}^{3}$ es cercana a $5 \mathrm{~cm}$. Esto puede verse en la distribución de tamaño de poros (Figura 1), donde los cilindros de $110 \mathrm{~cm}^{3}$ presentan una mayor proporción de poros de drenaje rápido (PDR) y una menor proporción de poros de agua útil (PAU) en relación a los cilindros de $250 \mathrm{~cm}^{3}$, aunque sin diferencias estadísticas significativas entre sí.

Al comparar los poros con aire y la permeabilidad de aire de los cilindros (Cuadro 2), se logró determinar que existen diferencias entre cilindros en las tensiones 10,20 y $30 \mathrm{hPa}$, observando que en el volumen menor $\left(\mathrm{Cil}_{110 \mathrm{~cm}^{3}}\right)$ el porcentaje de poros es significativamente más alto que el del volumen mayor $\left(\mathrm{Cil}_{250 \mathrm{~cm}} 3\right)$. A partir de tensiones mayores a $60 \mathrm{hPa}$ no se observaron diferencias estadísticamente significativas, lo que es relevante, ya que a partir de esta tensión se realizan una parte importante de los análisis físicos de suelo: i) para equilibrar muestras para análisis mecánico (p. ej. Vásquez et al., 2012), ii) para definir la capacidad de aire del suelo o volumen de poros de drenaje (p. ej. Seguel et al., 2013). Por otro lado, la determinación de permeabilidad de aire no se pudo realizar a tensiones menores de $30 \mathrm{hPa}$, debido a que el sistema poroso se encuentra saturado con agua, lo que genera una fase discontinua de aire que impide su flujo a través de los poros (Zúñiga et al., 2015). Las mediciones de $k_{a}$ no presentaron di-

Cuadro 1. Curva de retención de agua para dos volúmenes de muestreo.

Table 1. Water retention curve for two sampling volumes.

\begin{tabular}{|c|c|c|c|c|c|c|c|c|c|}
\hline \multirow{2}{*}{$\begin{array}{l}\psi \mathrm{m} \\
\mathrm{hPa}\end{array}$} & \multirow{2}{*}{$\begin{array}{c}\psi \mathrm{m} \\
\mathrm{pF}\end{array}$} & \multicolumn{4}{|c|}{$110 \mathrm{~cm}^{3}$} & \multicolumn{4}{|c|}{$250 \mathrm{~cm}^{3}$} \\
\hline & & $\% V$ & ES & cv $\%$ & & $\% V$ & ES & cv $\%$ & \\
\hline 0 & 0 & 52,00 & $\pm 1,24$ & 2,39 & $b$ & 55,45 & $\pm 0,62$ & 1,12 & a \\
\hline 10 & 1,00 & 46,33 & $\pm 1,30$ & 2,50 & e & 52,56 & $\pm 0,84$ & 1,52 & $b$ \\
\hline 20 & 1,30 & 45,27 & $\pm 1,28$ & 2,45 & ef & 51,17 & $\pm 0,88$ & 1,64 & bc \\
\hline 30 & 1,48 & 43,23 & $\pm 1,21$ & 2,33 & fgh & 49,21 & $\pm 0,91$ & 1,64 & $c d$ \\
\hline 60 & 1,78 & 42,49 & $\pm 1,18$ & 2,27 & ghi & 47,24 & $\pm 0,79$ & 1,43 & de \\
\hline 150 & 2,18 & 39,98 & $\pm 1,01$ & 1,95 & ij & 44,60 & $\pm 0,73$ & 1,31 & efg \\
\hline 330 & 2,52 & 38,94 & $\pm 0,96$ & 1,85 & jk & 42,68 & $\pm 0,66$ & 1,19 & fghi \\
\hline 500 & 2,70 & 37,19 & $\pm 0,84$ & 1,61 & k & 40,81 & $\pm 0,69$ & 1,24 & hij \\
\hline 15430 & 4,19 & 27,10 & $\pm 0,30$ & - & 1 & 25,46 & $\pm 0,30$ & - & I \\
\hline & & 54,75 & $\pm 0,73$ & & a & 57,49 & $\pm 0,40$ & & b \\
\hline
\end{tabular}

Nota: las letras distintas indican diferencias significativas ( $\mathrm{p} \leq 0,05)$ entre los distintos contenidos de agua y tamaños de cilindro. E.S.: error estándar. $\Psi_{\mathrm{m}}$ : potencial mátrico (tensión de agua) con su respectivo valor logarítmico (pF); contenido volumétrico (\%V). CV: Coeficiente de variación; Ep: Espacio poroso.

Note: the letters indicate significant differences $(\mathrm{p} \leq 0.05)$ between different water contents and cores sizes. E.S.: standard error. $\Psi$ : matric potential (water stress) with its respective logarithmic value (pF); Volumetric content (\% V). CV: Coefficient of variation. TP = total porosity. 
ferencias estadísticamente significativas entre los dos volúmenes a ningún nivel de tensión de agua aplicado en las muestras.

Al comparar la permeabilidad de aire de los dos volúmenes de muestreo (Figura 2) se puede observar que la permeabilidad medida a una misma tensión presentó la siguiente relación: $\mathrm{Cil}_{110 \mathrm{~cm}^{3}}>\mathrm{Cil}_{250 \mathrm{~cm}^{3}}$, en directa concordancia con la cantidad de poros llenos de aire, tal como lo demostró Martínez et al. (2016) para procesos de difusión en el suelo. Por lo mismo, a medida que aumenta la permeabilidad de aire esta relación se hace más cercana a la línea 1:1.

El incremento de la fracción de poros de aire y el aumento en la permeabilidad de aire (Figura 3 ), ha sido

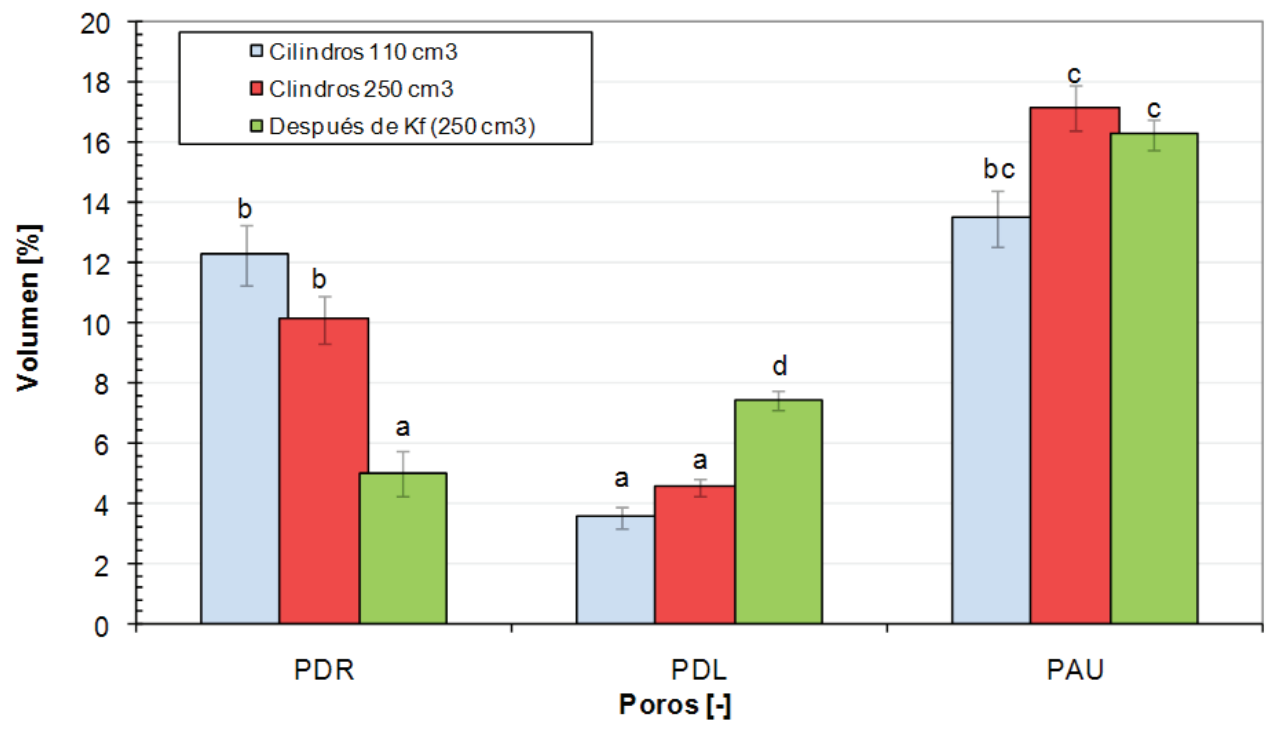

Figura 1. Distribución de tamaños de poros de distintos volúmenes de muestreo (para los tipos de poros, las barras indican \pm 1 E.S.; n: 8 en 110 y $250 \mathrm{~cm}^{3}$ y n: 14 en kf). PDR, poros de drenaje rápido ( $\left.>50 \mu \mathrm{m}\right)$; PDL: poros de drenaje lento (10-50 $\left.\mu \mathrm{m}\right)$; PAU, poros de agua útil $(0,2-10 \mu \mathrm{m})$.

Figure 1. Pore-size distribution for differentsamplingvolumes (for the type of pores, bars indicate \pm 1 E.S.; $n: 8$ in 110 and $250 \mathrm{~cm}^{3}$ $\mathrm{n}: 14 \mathrm{kf}) . \mathrm{wCP}=$ wide coarse pores $(>50 \mu \mathrm{m}) ; \mathrm{nCP}=$ narrow coarse pores $(10-50 \mu \mathrm{m}) ; \mathrm{MP}=$ middle pores $(0.2-10 \mu \mathrm{m})$.

Cuadro 2. Comparación de la fracción de poros llenos con aire y la permeabilidad de aire para dos volúmenes de muestreo.

Table 2. Comparison of air-filled porosity and air permeability for two sampling volumes.

\begin{tabular}{|c|c|c|c|c|c|c|c|c|c|c|c|c|}
\hline \multirow{3}{*}{$\begin{array}{l}\psi \mathrm{m} \\
\mathrm{hPa}\end{array}$} & \multicolumn{6}{|c|}{ 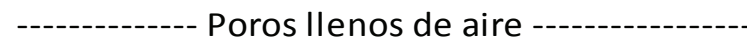 } & \multicolumn{6}{|c|}{--Permeabilidad de aire [log ${\left.\mu m^{2}\right]--}^{-}$} \\
\hline & \multicolumn{3}{|c|}{$110 \mathrm{~cm}^{3}$} & \multicolumn{3}{|c|}{$250 \mathrm{~cm}^{3}$} & \multicolumn{3}{|c|}{$110 \mathrm{~cm}^{3}$} & \multicolumn{3}{|c|}{$250 \mathrm{~cm}^{3}$} \\
\hline & $\% \mathbf{V}$ & ES & & $\% \mathbf{V}$ & ES & & $\mathbf{K a}$ & ES & & $\mathbf{K a}$ & ES & \\
\hline 10 & 8,42 & $\pm 1,01$ & $\mathrm{C}$ & 4,92 & $\pm 0,66$ a & a & - & & & & - & \\
\hline 20 & 9,49 & $\pm 1,05$ & $\mathrm{~cd}$ & 6,32 & $\pm 0,70$ & $\mathrm{~b}$ & - & & & & - & \\
\hline 30 & 11,52 & $\pm 1,00$ & defg & 8,27 & $\pm 0,73$ & c & 1,56 & $\pm 0,08$ & $a b$ & 1,33 & $\pm 0,14$ & a \\
\hline 60 & 12,27 & $\pm 0,98$ & efg & 10,25 & $\pm 0,67$ & de & 1,71 & $\pm 0,08$ & abcd & 1,65 & $\pm 0,12$ & $a b c$ \\
\hline 150 & 14,77 & $\pm 0,86$ & ghi & 12,88 & $\pm 0,60$ & fgh & 2,05 & $\pm 0,05$ & bcd & 1,96 & $\pm 0,11$ & abcd \\
\hline 330 & 15,82 & $\pm 0,82$ & hi & 14,81 & $\pm 0,57$ & ghi & 2,18 & $\pm 0,11$ & $\mathrm{~cd}$ & 2,09 & $\pm 0,11$ & $\mathrm{~cd}$ \\
\hline 500 & 17,56 & $\pm 0,76$ & $\mathrm{i}$ & 16,67 & $\pm 0,63$ & $\mathrm{i}$ & 2,28 & $\pm 0,13$ & $\mathrm{~d}$ & 2,26 & $\pm 0,12$ & d \\
\hline D.Vol. & 12,15 & $\pm 0,93$ & $a$ & 9,55 & $\pm 0,65$ & & 1,95 & $\pm 0,09$ & $a$ & 1,86 & $\pm 0,12$ & \\
\hline
\end{tabular}

Nota: - = no se determinó; letras distintas indican diferencias significativas $(\mathrm{p} \leq 0,05)$. E.S.: error estándar; curva de retención de agua (pF); permeabilidad de aire $\left(k_{a}\right)$; contenido volumétrico $(\% \mathrm{~V})$.

Note: - = not determined; Different letters indicate significant differences $(\mathrm{p} \leq 0.05)$. E.S.: standard error; Water retention curve (pF); Air permeability $\left(k_{a}\right)$; Volumetric content $(\% \mathrm{~V})$. 
observado por diversos autores (Ahuja et al., 1984; Ball et al., 1988; Roseberg y McCoy, 1990; Dörner y Horn, 2006; Resurrección et al., 2007). Además, el volumen de muestreo es indiferente cuando se correlacionan ambos parámetros, por ende, cambios en el monto de poros con aire se traducen en la misma proporción en cambios en la permeabilidad de aire, reflejado por que no existen diferencias significativas en la pendiente e intercepto de las curvas (Figura 3).

El volumen de muestreo no afectó a los índices de continuidad $M$ y $N$, ya que no se logró determinar que existieran diferencias estadísticas significativas. Sólo se observó un leve aumento en el volumen de los poros bloqueados $\left(\varepsilon_{b}\right)$ en las muestras de suelo obtenidas con

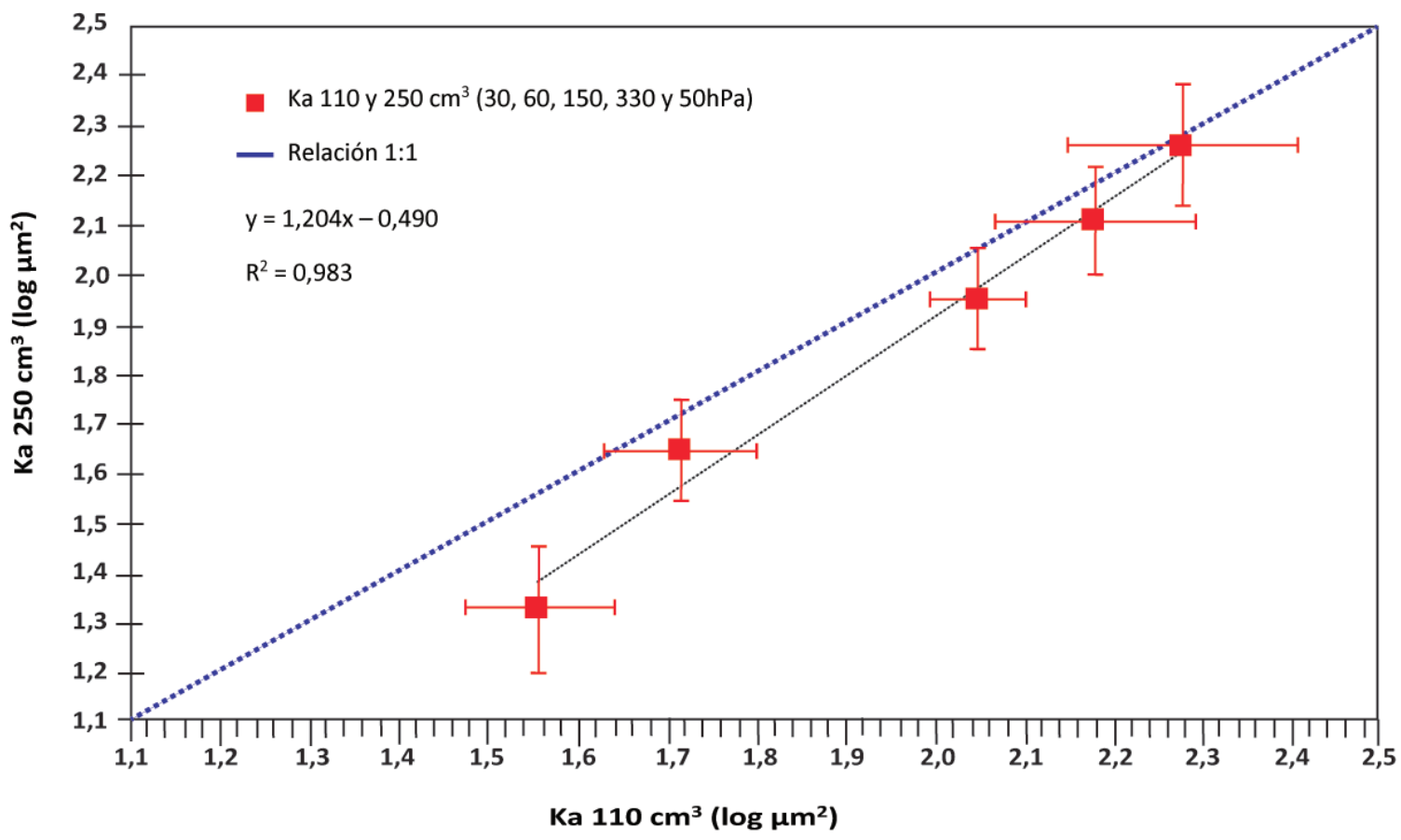

Figura 2. Comparación de la permeabilidad de aire $\left(k_{a}\right)$ en relación al volumen de muestreo (las barras indican \pm 1 E.S.; n: 8). Figure 2. Comparison of the air permeability $\left(k_{a}\right)$ in relation to the sampling volume (bars indicate \pm 1 E.S.; n: 8).

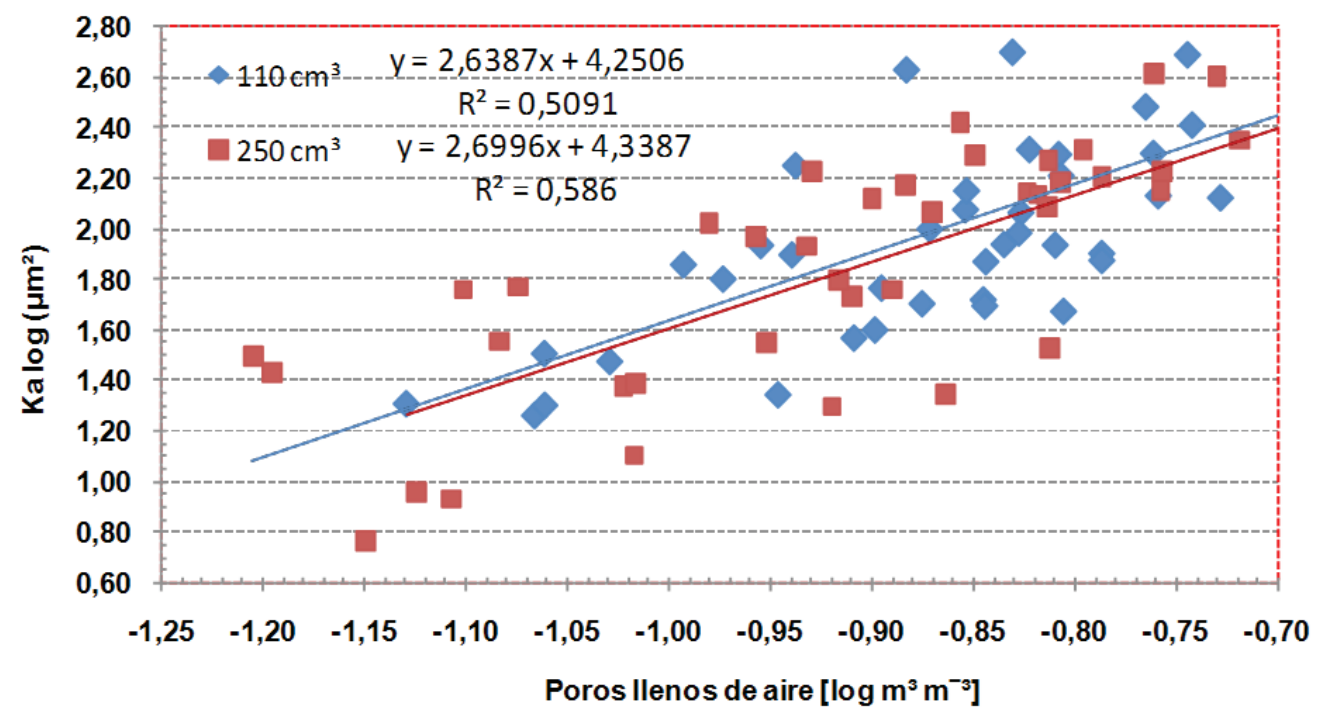

Figura 3. Permeabilidad de aire $\left(k_{a}\right)$ en función de los poros llenos con aire para los dos volúmenes de muestreo (las barras indican \pm 1 E.S.; n: 8).

Figure 3. Air permeability $\left(k_{a}\right)$ as function of the air-filled porosity for the two sampling volumes (bars indicate \pm 1 E.S., n: 8). 
los cilindros de $250 \mathrm{~cm}^{3}(0,69 \%)$ en comparación con cilindros de $110 \mathrm{~cm}^{3}$ (0,59\%).

El análisis general del volumen de muestreo determinó que el volumen de $110 \mathrm{~cm}^{3}$, es capaz de representar la funcionalidad del espacio poroso y la variabilidad existente en este suelo, pero no así el monto total de este. Aunque se registró una tendencia a tener un mayor espacio poroso y permeabilidad de aire a tensiones mayores a $60 \mathrm{hPa}$, los valores determinados no reflejan diferencias estadísticas en la continuidad del medio poroso entre los dos volúmenes de muestreo, por lo tanto es viable usar un volumen de $110 \mathrm{~cm}^{3}$ para caracterizar la heterogeneidad del sistema poroso del suelo en parámetros de funcionalidad.

Comparación de la permeabilidad de aire con la conductividad hidráulica y sus efectos sobre el sistema poroso

La permeabilidad al aire $\left(k_{a}\right)$ y la conductividad hidráulica (kf) son parámetros usados para determinar la funcionalidad del sistema poroso (Dörner, 2005; Quiroz, 2005; Zúñiga et al., 2014). Ambas propiedades han sido utilizadas para modelar el flujo de agua en el suelo en estado saturado y no saturado (Hartge y Horn,
2009). La Figura 4 presenta la relación entre kf (medición a las 24 horas, para determinar si existen cambios en kf y en el espacio poroso por efecto de un flujo constante de agua a través del suelo). Existe una alta relación entre la permeabilidad de aire y la conductividad hidráulica saturada (Poulsen et al., 2007), principalmente con las mediciones de ka realizadas a -60 y -150 hPa, ya que sería a través de estos poros donde se producirían principalmente los flujos de agua en el suelo, asociado a los mayores diámetros de poros.

Los flujos de agua y aire transitarían por los mismos poros en el suelo y las diferencias se deben a las características físicas del fluido, ya que el aire posee un roce mínimo en los bordes de los poros, lo que no es así para el flujo del agua (Sumner, 2000). Esto concuerda con una disminución en $\mathrm{kf}$ de $-3,19$ a -3,59 $\left(\log \mathrm{cm} \mathrm{s}^{-1}\right)$ entre la primera hora y pasado 24 horas de flujo continuo (datos no presentados), provocando una disminución del contenido de PDR (Figura 1), los cuales están directamente relacionados con el flujo de ambos fluidos. Por otro lado, aumentaron los poros de drenaje lento (PDL) y disminuyeron levemente los PAU, aunque sin diferencias estadísticas significativas en estos últimos. Ellies et al. (1997), propusieron que kf puede ser usado como una herramienta para evaluar la estabilidad es-
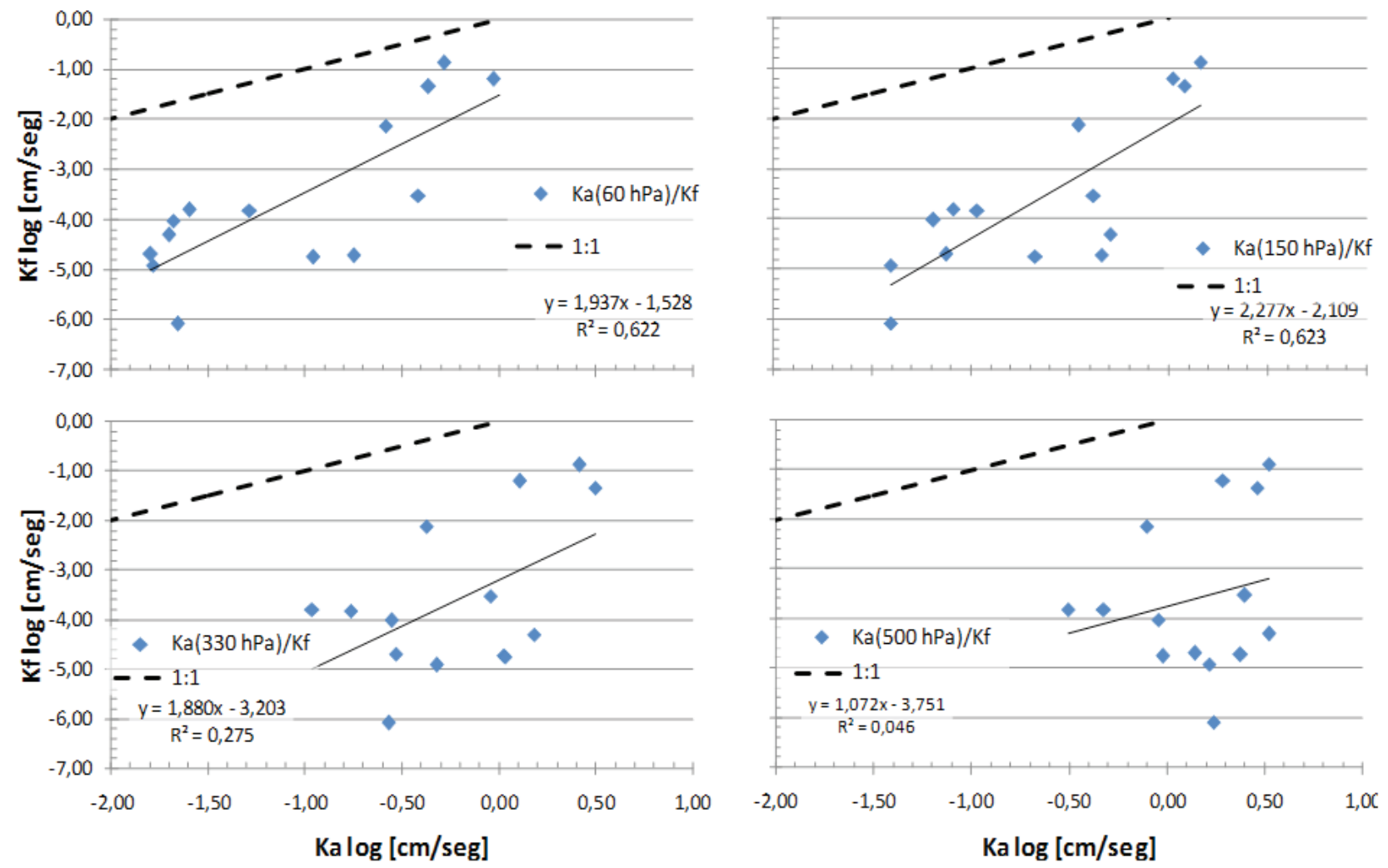

Figura 4. Relación entre la conductividad hidráulica saturada (kf) y la permeabilidad de aire (ka) medida a distintas tensiones. Figure 4. Relationship between saturated hydraulic conductivity (kf) and air permeability (ka) measured at different tensions. 
tructural del suelo, generándose cambios significativos en el flujo de agua después de realizar una medición constante de 100 horas. Esto también fue analizado para distintos Andisoles por Dörner et al. (2010), quienes concluyeron que las mediciones de $k_{a}$ comparadas con kf resultan más fáciles de controlar, asegurando un flujo laminar en los poros del suelo, no así en kf, donde la metodología usada puede generar flujos turbulentos, aumentando las pérdidas de carga por roce y disminuyendo el flujo de agua a través del suelo.

La permeabilidad de aire y los poros llenos con aire siguen presentando una alta correlación posterior a la medición de kf, aunque disminuye en relación a las muestras no alteradas por el efecto del flujo constante del agua (Figura 5). La permeabilidad de aire es estadísticamente diferente $(p \leq 0,05)$ en los volúmenes de 250 $\mathrm{cm}^{3}$ evaluados después de kf en las tensiones de -60, y $-150 \mathrm{hPa}$. Para los poros llenos de aire no se encontraron diferencias estadísticas significativas a partir de 60 $\mathrm{hPa}$ de tensión. La pérdida de porosidad afectó a la permeabilidad de aire, lo que supondría que los poros que se perdieron cumplen un rol funcional dentro del suelo, y que por efecto del flujo constante de agua se alteró parte la estructura, cambiando la funcionalidad de los poros. Por otro lado, los cambios en el volumen y distribución de los poros provocó un aumento en el índice de poros bloqueados (Figura 6) probablemente por el arrastre de partículas por el agua, lo que contribuyó a la disminución del volumen de PDR y $k_{a}$ en el suelo. Lo anterior es relevante, ya que se con la disminución de los PDR (Figura 1) alcanzaría un nivel de macroporos similares a los de un suelo que presenta problemas de compactación, reduciendo la capacidad de trasmisión de agua y aire (Horn y Fleige, 2009).

Los parámetros de continuidad en el sistema poroso (Figura 7) presentan diferencias estadísticas en $k_{1}$ sólo en las mediciones realizadas después de determinar kf entre las tensiones de 150 y $500 \mathrm{hPa}$, en el volumen de $250 \mathrm{~cm}^{3}$. Para $k_{2}$ se determinó un aumento de la continuidad a $60 \mathrm{hPa}$, encontrando valores significativamente mayores a dicha tensión después de kf, lo que supone que a pesar de una pérdida del volumen de poros por efecto del roce durante el flujo de agua, los poros que no se bloquearon y que participan en la transmisión del aire a esta tensión son más continuos y menos tortuosos. En este caso, además, se presenta una alta variabilidad, debido a la inestabilidad y alteración del sistema poroso $>50 \mu \mathrm{m}$ por efecto del flujo continuo de agua. Sin embargo, en los parámetros de continuidad $M$ y $N$ no se logró determinar diferencias estadísticas entre los tres tratamientos.

\section{CONCLUSIONES}

Las distintas fracciones del espacio poroso del suelo puede ser descrito de forma representativa tanto en su volumen como en su funcionalidad con un volumen de muestreo de $110 \mathrm{~cm}^{3}$ solo a partir de una tensión de agua de $60 \mathrm{hPa}$. Lo anterior es relevante, ya que esta tensión de agua permite el drenaje de los poros que representa la capacidad de aire del suelo o el volumen de poros de drenaje, poros que son susceptibles al efecto del manejo del suelo. Entre 0 y $30 \mathrm{hPa}$ de tensión se observaron diferencias en el volumen de

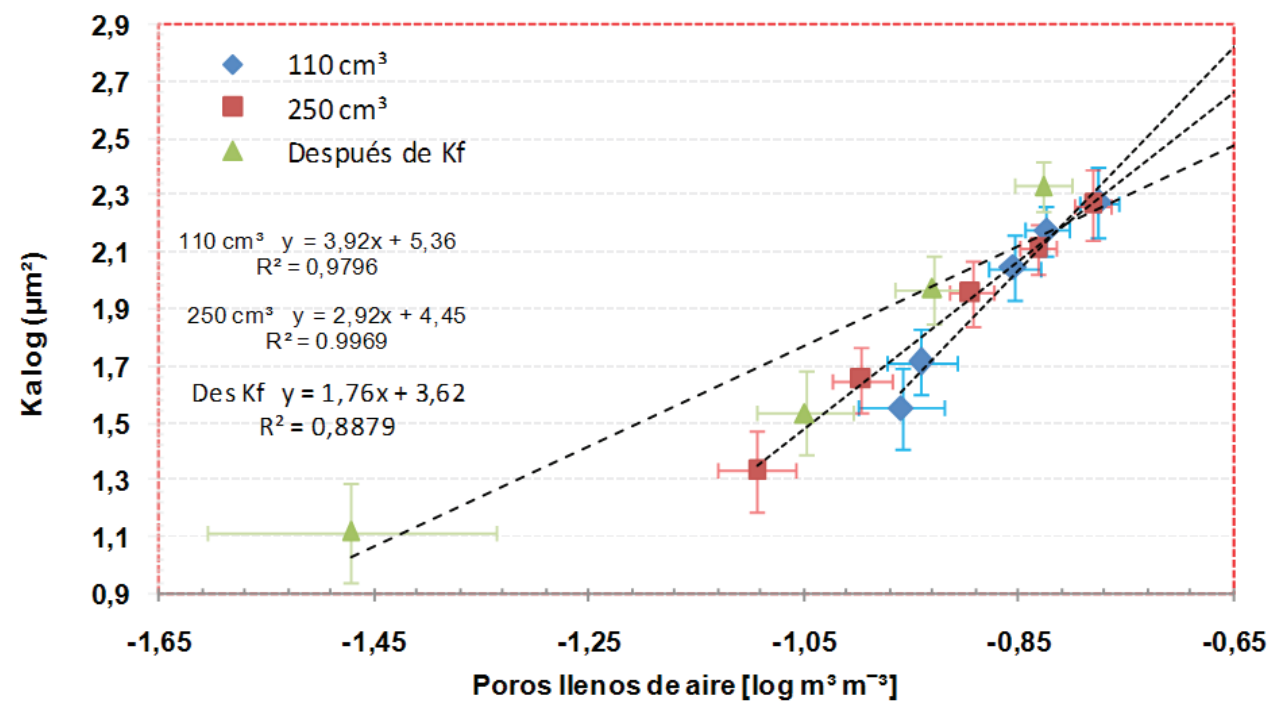

Figura 5. Permeabilidad de aire en función de los poros llenos con aire para los dos volúmenes de muestreo y las mediciones realizadas después de kf (las barras indican \pm 1 E.S.; n: 8 en 110 y $250 \mathrm{~cm}^{3}$ y n: 14 en kf).

Figure 5. Air permeability as function of the air-filled porosity for the two sampling volumes and measurements made after kf (bars indicate \pm 1 E.S.; n: 8 in 110 and $250 \mathrm{~cm}^{3}$ and n: $14 \mathrm{kf}$ ). 
muestreo, cuyas implicancias deben ser mayormente investigadas, para una adecuada representación de los poros del suelo.

El flujo saturado de agua a través de los poros del suelo generó una pérdida de estructura que afectó negativamente el volumen de macroporos (poros de drenaje) y su capacidad de conducción. Además, aumentó la cantidad de poros bloqueados y continuidad del medio poroso para los índices $k_{1}$ y $k_{2}$ principalmente a $60 \mathrm{hPa}$, lo que indicaría que parte del sistema poroso pierde su funcionalidad producto de la obstrucción por partículas que son desplazadas por el flujo saturado de agua.

\section{AGRADECIMIENTOS}

Los autores agradecen el financiamiento de este trabajo por parte del Instituto de Ingeniería Agraria y Suelos de la Universidad Austral de Chile.

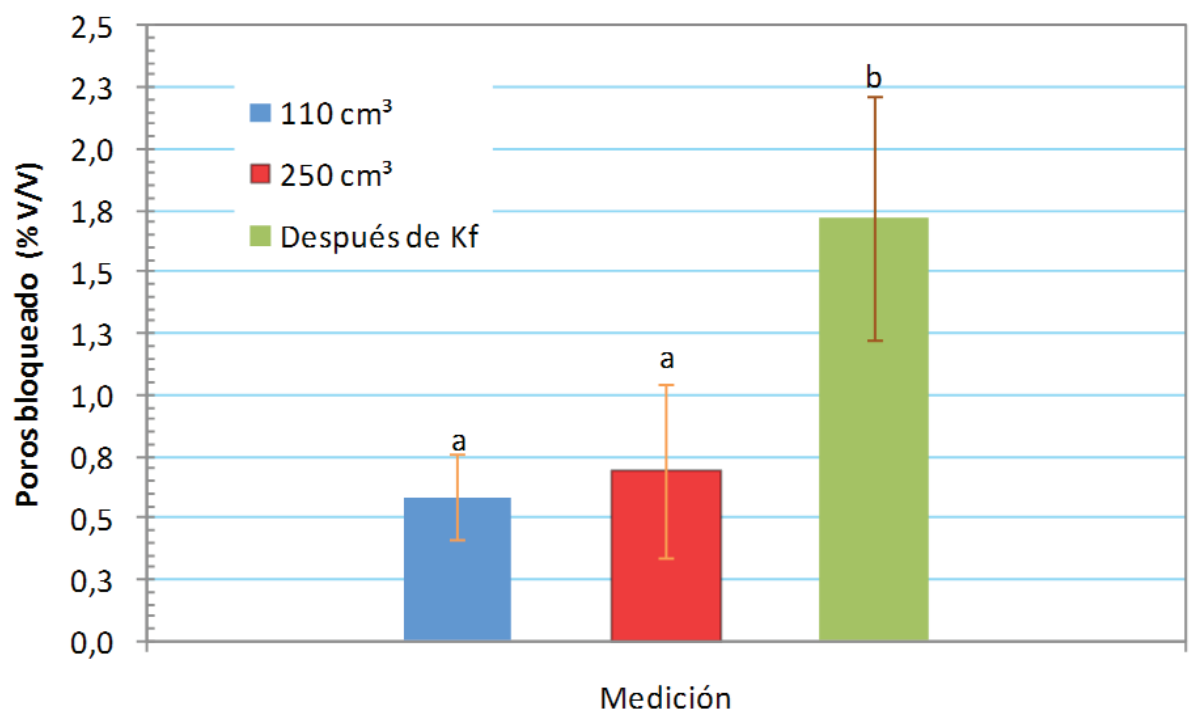

Figura 6. Volumen de poros bloqueados para los dos volúmenes de muestreo y las mediciones realizadas después de kf (las barras indican \pm 1 E.S.; n: 8 en 110 y $250 \mathrm{~cm}^{3}$ y n: 14 en kf).

Figure 6. Volume of blocked porosity for the two sampling volumes and measurements made after kf (bars indicate \pm 1 E.S.; $\mathrm{n}: 8$ in 110 and $250 \mathrm{~cm}^{3}$ and $\mathrm{n}: 14 \mathrm{kf}$ ).
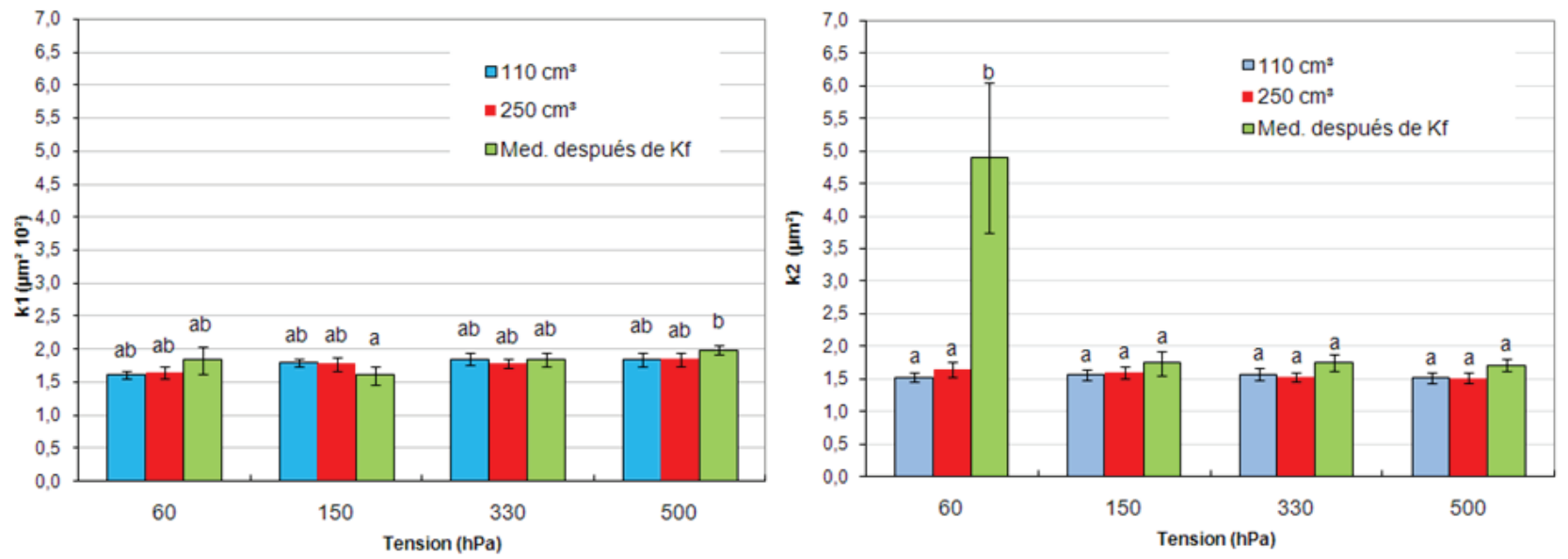

Figura 7. Índices de continuidad $k_{1}$ y $k_{2}$ de los distintos volúmenes de muestreo y las mediciones realizadas después de kf (las barras indican \pm 1 E.S.; n: 8 para mediciones de 110 y $250 \mathrm{~cm}^{3}$ y n: 14 para mediciones después de kf).

Figure 7. Continuity indexes $k_{1}$ and $k_{2}$ of the different sampling volumes and measurements made after kf (bars indicate \pm 1 E.S.; $\mathrm{n}$ : 8 for measurements of 110 to $250 \mathrm{~cm}^{3} \mathrm{n}: 14$ for measurements after kf). 


\section{REFERENCIAS}

Ahuja, L., Naney, J., Green, R., Nielsen, D., 1984. Macroporosity to characterize spatial variability of hydraulic conductivity and effects of land management. Soil Science Society of American Journal 48, 699-702.

Ball, B., O’Sullivan, M., Hunter, R., 1988. Gas diffusion, fluid flow and derived pore continuity indices in relation to vehicle traffic and tillage. Journal of Soil Science 39, 327339.

Bartoli, F., Genevois-Gomendy, V., Royer, J., Niquet, S., Vivier, H., Grayson, R., 2005. A multiscale study of silty soil structure. European Journal of Soil Science 56, 207-223.

Clifford, K., Stephen, W., 2006. Gas transport in porous media. Springer, New York.

Centro de Información de Recursos Naturales (CIREN), 2003. Estudio Agrológico X Región. Publicación N 123, Santiago, Chile.

Dexter, A.R., 2004. Soil physical quality. Part I. Theory, effects of soil texture, density, and organic matter, and effect on root growth. Geoderma 120, 201-214.

Dietrich, P., Helmig, R., Sauter, M., Hötzl, H., Köngeter, J., Teutsch, G., 2005. Flow and Transport in Fractured Porous Media. Springer, New York.

Dörner, J., 2005. Anisotropie von Bodenstrukturen und Porenfunktionen in Böden und deren Auswirkungen auf Transportprozesse im gesättigten und ungesättigten Zustand (Doctoral dissertation, Christian-Albrechts Universität Kiel). Nr. 68, 182 p. ISBN: 0933-680 X.

Dörner, J., Horn, R., 2006. Anisotropy of pore functions in structured Stagnic Luvisols in the Weichselian moraine region in N Germany. Journal Plant Nutrition of Soil Science 169, 213-220.

Dörner, J., Dec, D., 2007. La permeabilidad del aire y conductividad hidráulica saturada como herramienta para la caracterización funcional de los poros del suelo. Revista de la Ciencia del Suelo y Nutrición Vegetal 7(2), 1-13.

Dörner, J., Sandoval, P., Dec, D., 2010. The role of soil structure on the pore functionality of an ultisol. Journal of Soil Science and Plant Nutrition 10(4), 495-508.

Dörner, J., Dec, D., Feest, E., Vásquez, N., Díaz, M., 2012. Dynamics of soil structure and pore functions of a volcanic ash soil under tillage. Soil and Tillage Research 125, 52-60.

Ellies, A., Grez, R., Ramírez, C., 1997. La conductividad hidráulica en fase saturada como herramienta para el diagnóstico de la estructura del suelo. Agro Sur 25(1), 51-56.

Fuentes, I., Casanova, M., Seguel, O., Padarian, J., Nájera, F., Salazar, 0., 2015. Preferential flow paths in two alluvial soils with long-term additions of pig slurry in the Mediterranean zone of Chile. Soil Research 53(4), 433-447.

Hartge, K., Horn, R., 1991. Einführung in die dodenphysik. Enke. Stuttgart. Germany.

Hartge, R., Horn, R., 2009. Die physikalische Untersuchung von Bóden. Praxis Messmethoden Auswertung. 4. vollst. Überarbeitete Auflage. Schweizerbart Vorlage, Stuttgart.

Hillel, D., 1982. Introduction to Soil Physics. Academic Press, USA.

Hillel, D., 1998. Environmental Soil Physics. Academic Press, San Diego, California.

Hopmans, J., van Genuchten, M., 2005. Vadose zone: hydrological process, in: Hillel, D. (Ed.), Encyclopedia of Soils in the Environment. Elsevier, The Netherlands, pp. 209216.

Horn, R., Smucker, A., 2005. Structure formation and its consequences for gas and water transport in unsaturated arable and forest soils. Soil and Tillage Research 82, 5-14.

Horn, R., Fleige, H., 2009. Risk assessment of subsoil compaction for arable soils in Northwest Germany at farm scale. Soil and Tillage Research 102, 201-208.

Hunt, A., 2005. Percolation theory for flow in porous media. Springer, New York, USA.

Iversen, B., Moldrup, P., Schjønning, P., Loll, P., 2001. Air and water permeability in differently textured soils at two measurement scales. Soil Science 166(10), 643-659.

Lal, R., Manoj, S., Shukla, A., 2004. Principles of Soil Physics. CRC Press, USA.

Leiva, C., 2009. Caracterización del monto, funcionalidad y evolución del sistema poroso de un Palehumult al ser sometido a ciclos de formación de estructura. Tesis Ingeniero Agrónomo, Universidad Austral de Chile. 77 p.

Martínez, I., Chevert, A., Weisskopfa, P., Sturny, W.G., Reka, J., Keller, T., 2016. Two decades of no-till in the Oberacker long-term field experiment: Part II. Soil porosity and gas transport parameters. Soil and Tillage Research 163, 130-140.

Noellemeyer, E., Fernández, R., Quiroga, A., 2013. Crop and tillage effects on water productivity of dryland agriculture in Argentina. Agriculture 3, 1-11.

Pagenkemper, S., Puschmann, D.U., Peth, S., Horn, R., 2014. Investigation of time dependent development of soil structure and formation of macropore networks as affected by various precrop species. International Soil and Water Conservation Research 2(2), 51-66.

Poulsen, T., Moldrup, P., Mogensen, M., Jacobsen, O., 2007. Solute diffusion analogy model for predicting unsaturated hydraulic conductivity in repacked soil. Soil Science 172(2), 101-107.

Quiroz, C., 2005. Movimiento de agua en suelos andisoles sujetos a distintos usos. Tesis Ingeniero Agrónomo, Universidad Austral de Chile. 61p.

Rawls, W., Pachepsky, Y., 2002. Soil consistence and structure as predictors of water retention. Soil Science and Society of American Journal 66, 1115-1126.

Resurrección, A., Kawamoto, K., Komatsu, T., Moldrup, P., Sato, K., Rolston, D., 2007. Gas diffusivity and air permeability in a volcanic ash soil profile: effects of organic matter and water retention. Soil Science 172(6), 432-443.

Roseberg, R., McCoy, E., 1990. Measurement of soil air permeability. Soil Science Society of American Journal 54, 969-974.

Saxton, K., Rawls, W., 2003. Soil water characteristic estimates by texture and organic matter for hydrologic solutions. Soil Science Society of American Journal 70, 1569-1578.

Seguel, O., Sagardía, S., Casanova, M., 2013. Efecto del exudado de Chaitophorus leucomelas sobre las propiedades hidráulicas del suelo. Agro Sur 41(2), 1-7.

Sumner, M., 2000. Handbook of Soil Science. CRC Press. Florida, USA.

Uribe, J.M., Cabrera, R., De La Fuente, A., Paneque, M., 2012. Atlas Bioclimático de Chile. CORFO, Universidad de Chile., Santiago, Chile.

Vafai, K., 2005. Handbook of Porous Media. Taylor \& Francis. 
New York, USA.

Vásquez, N., Salazar, F., Dörner, J., 2012. Variabilidad temporal de las propiedades físico-mecánicas de un suelo derivado de cenizas volcánicas bajo labranza convencional. Agro Sur 40(3), 1-13.

Zúñiga, F., Dec, D., Valle, S.R., Dörner, J., MacDonald, R., 2014. Estabilidad estructural de un Andisol (Typic Durudand) bajo bosque nativo y pradera en el Sur de Chile. Agro Sur 42(3), 55-66.

Zúñiga, F., Ivelic-Sáez, J., López, I., Huygens, D., Dörner, J., 2015. Temporal dynamics of the physical quality of an Andisol under a grazing system subjected to different pasture improvement strategies. Soil and Tillage Research 145, 233-241. 
